\title{
ON FINITE SIMPLE SEMIGROUPS
}

\author{
by JORGE ALMEIDA
}

(Received 27th March 1989)

\begin{abstract}
Implicit operations (new operations commuting with all old homomorphisms) on pseudovarieties have been shown to play an important role in the study of these classes. They may be used to axiomatize subpseudovariaties and to describe recognizable subsets of (relatively) free objects. This paper presents a case study for the pseudovariety CS consisting of all finite simple semigroups. Based on a result of profinite group theory, a structural description of semigroups of implicit operations on finite simple semigroups is used to deduce that $\mathrm{CS}$ is join-irreducible.
\end{abstract}

1980 Mathematics subject classification (1985 Revision): 20M07, $20 \mathrm{M} 35$.

\section{Introduction}

Following the publication of Eilenberg's treatise [12] which set up an apropriate framework for the study and applications of finite semigroups and monoids, there has been an increasing interest in pseudovarieties of these types of algebraic structures (cf. Lallement [17] and Pin [18]). However, in spite of the obvious connections with standard constructions of universal algebra (cf. Eilenberg and Schützenberger [13]), it took several years before they started to be explored in a systematic manner.

A pseudovariety is a class of finite algebras of the same type which is closed under the formation of homomorphic images, subalgebras and finitary direct products. There are several interesting and useful alternative ways of describing pseudovarieties (see, e.g., [2]). Among these, the most significant appear to be the correspondence with the socalled varieties (or streams) of rational languages obtained by Eilenberg [12], the link with the theory of Birkhoff's varieties via "generalized varieties" (upper directed unions of varieties) established by Ash [7], and the definition of pseudovarieties by means of "pseudoidentities" as given by Reiterman [20]. Reiterman's approach consists in considering new algebraic ("implicit") operations which do not conflict with the old homomorphisms. He then shows that, fixing the arity, they form a compact metric space in a reasonable way and uses this property to prove that pseudovarieties are defined by formal equalities of implicit operations. Banaschewski [8] and the author $[1,3,4]$ have generalized and given alternative treatments of this result.

In this paper, we propose the study of a pseudovariety of semigroups which, for their algebraic properties, have always deserved a lot of attention in the general theory of semigroups, namely completely simple semigroups. The first significant structure theorem about semigroups deals precisely with completely simple semigroups (cf. 
Lallement [17]) For the case of finite semigroups, the property characterizing completely simple semigroups is equivalent to the semigroup being simple, i.e., having no proper non-empty ideals. Based on Clifford's description [10] of free completely simple semigroups, we describe the implicit operations on the pseudovariety CS of all finite simple semigroups and we use it to describe the subsets of a free semigroup over a finite alphabet which may be recognized by a homomorphism into a member of CS. As a different kind of application, we study the join-irreducibility of CS, showing that it is a consequence of a property of groups of implicit operations on finite groups.

\section{Preliminaries}

Let $\mathbf{V}$ be a pseudovariety of semigroups. The set $\bar{\Omega}_{n} \mathbf{V}$ of all $n$-ary implicit operations on $\mathrm{V}$ consists of all $\mathrm{V}$-indexed families $\pi=\left(\pi_{A}\right)_{A \in \mathrm{V}}$ such that:

(i) $\pi_{A}: A^{n} \rightarrow A$ is a function;

(ii) if $A, B \in \mathbf{V}$ and $h: A \rightarrow B$ is a homomorphism, then $h \circ \pi_{A}=\pi_{B} \circ h^{n}$.

Define a product on $\bar{\Omega}_{n} \mathbf{V}$ pointwise: for $\pi, \rho \in \bar{\Omega}_{n} \mathbf{V}, A \in \mathbf{V}$ and $v \in A^{n}$,

$$
(\pi \rho)_{A}(v)=\pi_{A}(v) \rho_{A}(v) .
$$

Then obviously $\bar{\Omega}_{n} \mathrm{~V}$ is a semigroup under this operation. Represent by $x_{i}$ the element of $\bar{\Omega}_{n} \mathbf{V}$ such that $\left(x_{i}\right)_{A}: A^{n} \rightarrow A$ is the projection onto the $i$ th component $(i=1, \ldots, n)$. Then, as it is well-known [9], the subsemigroup $\Omega_{n} \mathbf{V}$ of $\bar{\Omega}_{n} \mathbf{V}$ generated by $\left\{x_{1}, \ldots, x_{n}\right\}$ is freely generated by this set in the (Birkhoff) variety generated by $\mathrm{V}$. The members of $\Omega_{n} \mathrm{~V}$ are called $n$-ary explicit operations on $\mathbf{V}$. An important and common example of an implicit operation which is not explicit on most pseudovarieties of semigroups is the unary operation $x^{\omega}$ : for a finite semigroup $S$ and $s \in S$, take $s^{\omega}$ to be the unique idempotent in $S$ which is a power of $s$. We will also consider the unary implicit operation $x^{\omega-1}$ : with $S$ and $s$ as before, if $s^{\omega}=s^{r}$, let $s^{\omega-1}=s^{2 r-1}$.

We endow $\bar{\Omega}_{n} \mathbf{V}$ with the initial topology for the homomorphisms into members of $\mathbf{V}$, which are viewed as discrete topological spaces. Then one may show that $\bar{\Omega}_{n} \mathbf{V}$ is a topological semigroup which is compact zero-dimensional having $\Omega_{n} \mathbf{V}$ as a dense subset. Moreover, for a finite semigroup $S, S$ lies in $V$ if and only if there is a positive integer $n$ and a continuous onto homomorphism $\bar{\Omega}_{n} \mathrm{~V} \rightarrow S$. For details see $[1,4,8,20]$.

Say that a subset $L$ of $\Omega_{n} \mathbf{V}$ is $\mathbf{V}$-recognizable if there is a homomorphism $\phi: \Omega_{n} \mathbf{V} \rightarrow S \in \mathbf{V}$ and $K \subseteq S$ such that $L=\phi^{-1} K$. These subsets are intimately related with the structure of $\bar{\Omega}_{n} \mathbf{V}$. Indeed, a subset $L$ of $\Omega_{n} \mathbf{V}$ is $\mathbf{V}$-recognizable if and only if $L=K \cap \Omega_{n} V$ for some clopen (i.e., closed and open) subset $K$ of $\bar{\Omega}_{n} \mathbf{V}$ [3].

A V-pseudoidentity is a formal equality $\pi=\rho$ between implicit operations on $\mathbf{V}$ of the same arity. The $V$-pseudoidentity $\pi=\rho$ is said to hold in $S \in V$ if $\pi_{s}=\rho_{s}$. In the following we will always refer to "pseudoidentities" meaning "pseudoidentities over the class of all finite semigroups". For a set $\Sigma$ of pseudoidentities, $\llbracket \Sigma \rrbracket$ denotes the class of all finite semigroups in which all pseudoidenities from $\Sigma$ hold. Certainly every class of the form $\llbracket \Sigma \rrbracket$ is a pseudovariety. The converse was proved by Reiterman [20]. For example, the 
class CS of all finite completely simple semigroups and the class $\mathbf{G}$ of all finite groups are given by

$$
\begin{gathered}
\mathbf{C S}=\llbracket(x y x)^{\omega}=x^{\omega}, x^{\omega+1}=x \rrbracket \\
\mathbf{G}=\llbracket x^{\omega}=1 \rrbracket
\end{gathered}
$$

where $x^{\omega+1}$ represents $x x^{\omega}$ and $x^{\omega}=1$ abbreviates $x^{\omega} y=y x^{\omega}=y$.

Recall that completely simple semigroups may be represented as Rees matrix semigroups, a result sometimes referred to as the Rees-Suschkewitsch theorem. We will use the following notation for Rees matrix semigroups [11]: given a group $G$, sets $\Lambda$ and $I$, and a $I \times \Lambda$-matrix $P$ with entries in $G$,

$$
\mathscr{M}(G ; \Lambda, I ; P)
$$

denotes the semigroup whose elements are all triples of the form $(\lambda, g, i)$ with $\lambda \in \Lambda, g \in G$, and $i \in I$, in which multiplication is given by

$$
(\lambda, g, i)(\mu, h, j)=\left(\lambda, g p_{i \mu} h, j\right)
$$

where $p_{i \mu}$ is the entry of $P$ in row $i$ and column $\mu$. We will always distinguish an element of $\Lambda$ and an element of $I$ which will be taken to be 1 in both cases, and we assume that the matrix $P$ is normalized along row 1 and column 1: if either $i=1$ or $\lambda=1$, then $p_{i \lambda}=1$. Then $g \mapsto(1, g, 1)$ defines an embedding of $G$ in $\mathscr{M}(G ; \Lambda, I ; P)$ so that we usually identify $G$ with the subgroup $\{1\} \times G \times\{1\}$ of $\mathscr{M}(G ; \Lambda, I ; P)$.

In case $G, \Lambda$ and $I$ are also viewed as topological spaces, we endow the set $\mathscr{M}(G ; \Lambda, I ; P)$ with the product topology. Since we are only interested in the case where $\Lambda$ and $I$ are discrete spaces, we then have that $\mathscr{M}(G ; \Lambda, I ; P)$ is a topological semigroup.

Lemma 2.1. The semigroup $\bar{\Omega}_{n} \mathrm{CS}$ is completely simple and it has $n \mathscr{R}$-classes and $n$ $\mathscr{L}$-classes.

Proof. Let $\pi, \rho \in \bar{\Omega}_{n} \mathbf{C S}$. Then the pseudoidentities.

$$
\begin{gathered}
\pi(\pi \rho \pi)^{\omega-1} \pi \rho \pi=\pi \\
\pi^{\omega+1}=\pi
\end{gathered}
$$

hold in CS and, therefore, the corresponding equalities of implicit operations hold in $\bar{\Omega}_{n} \mathrm{CS}$. Since $\pi \pi^{\omega-1}=\pi^{\omega-1} \pi=\pi^{\omega}$ and $\pi^{\omega}$ is an idempotent, from the second equality above we deduce that $\pi$ lies in the group with idempotent $\pi^{\omega}$. But, by the first equality, the idempotents in $\bar{\Omega}_{n}$ CS form a rectangular band. Hence $\bar{\Omega}_{n}$ CS is completely simple.

If $w \in \Omega_{n} C S$ starts with $x_{i}$ and ends with $x_{j}$, then $w^{\omega}=\left(x_{i} x_{j}\right)^{\omega}$ in any member of CS and so $x_{j} \mathscr{L}_{w} \mathscr{R} x_{i}$ in $\Omega_{n} \mathrm{CS}$. Moreover, since CS contains all finite rectangular bands, for $i \neq j, x_{i}$ and $x_{j}$ do not lie in the same $\mathscr{R}$ - or $\mathscr{L}$-class. 
If $\pi=\lim w_{k}$ in $\Omega_{n}$ CS with $w_{k} \in \Omega_{n}$ CS, we may assume that all $w_{k}$ start with the same $x_{i}$ and end with the same $x_{j}$. Let $x_{i}=w_{k} \rho_{k}$ with $\rho_{k} \in \bar{\Omega}_{n}$ CS. Since $\bar{\Omega}_{n}$ CS is compact, we may assume that $\lim \rho_{k}=\rho$. By continuity of the product operation in $\bar{\Omega}_{n} \mathbf{C S}, x_{i}=\pi \rho$. Hence $x_{i} \leqq \pi$. Analogous arguments show that $x_{j} \mathscr{L} \pi \mathscr{R} x_{i}$. Hence $\bar{\Omega}_{n}$ CS has $n \mathscr{R}$-classes and $n \mathscr{L}$-classes.

Since finite completely simple semigroups do not satisfy any nontrivial semigroup identities (because finite groups do not, cf. Evans [14]) $\Omega_{n}$ CS is the free semigroup on $\left\{x_{1}, \ldots, x_{n}\right\}$. This is not, of course, in contradiction with Clifford's characterization [10] of the free completely simple semigroup, since we are not considering here the same algebraic type. Nevertheless, the free completely simple semigroup on $n$ generators is the subalgebra of type $(2,1)$ of $\Omega_{n}$ CS generated by $\left(x_{1}, \ldots, x_{n}\right\}$, where the unary operation is precisely $x^{\omega-1}$. Thus, in view of Clifford's results, the above lemma and our structure theorem for $\bar{\Omega}_{n}$ CS in the next section should be no surprise, and so the theorem could perhaps be derived from these results. However, a more direct approach as the one adopted here seems more appropriate, as it openly shows the interaction between group implicit operations and implicit operations on CS.

\section{Implicit operations on simple semigroups}

In the following, we fix a positive integer $n$ and we take $m=n+(n-1)^{2}$. We will refer to the canonical generators of the group $\bar{\Omega}_{m} G$ by $x_{1}, \ldots, x_{n}$ (projections to the first $n$ components) and $y_{i j}$ with $i, j=2, \ldots, n$ (projections to the remaining $(n-1)^{2}$ components).

Consider the mapping

$$
\phi: \bar{\Omega}_{n} \mathrm{CS} \rightarrow \bar{\Omega}_{m} \mathbf{G}
$$

defined by

$$
\left(1, \phi(\pi)_{A}\left[a_{1}, \ldots, a_{n},\left(a_{i j}\right)\right], 1\right)=(1,1,1) \pi_{S}\left[\left(1, a_{1}, 1\right), \ldots,\left(n, a_{n}, n\right)\right](1,1,1)
$$

for $A \in \mathbf{G}$ and $a_{k}, a_{i j} \in A$ where $S=\mathscr{M}(A ; n, n ; P)$ with $P$ obtained from the matrix $\left(a_{i j}\right)$ by adding a first row and a first column of 1 's.

Lemma 3.1. The mapping $\phi$ is a well-defined continuous homomorphism.

Proof. Suppose that $h: A \rightarrow B$ is a homomorphism with $A, B \in \mathbf{G}$ and let $a_{k}, a_{i j} \in A$ $(k=1, \ldots, n ; i, j=2, \ldots, n)$. Then

$$
\begin{gathered}
h^{\prime}: \mathscr{M}(A ; n, n ; P) \rightarrow \mathscr{M}(B ; n, n ; Q) \\
(i, a, j) \mapsto(i, h(a), j)
\end{gathered}
$$


defines a homomorphism, where $P$ and $Q$ are defined as above for the matrices $\left(a_{i j}\right)$ and $\left(h\left(a_{i j}\right)\right)$ respectively, since

$$
h^{\prime}\left[(i, a, j)\left(k, a^{\prime}, l\right)\right]=\left(i, h\left(a a_{j k} a^{\prime}\right), l\right)=h^{\prime}(i, a, j) h^{\prime}\left(k, a^{\prime}, l\right) .
$$

Hence, for $\pi \in \bar{\Omega}_{n} \mathbf{C S}$,

$$
\phi(\pi)_{B} \circ h^{n}=\left.\pi_{T} \circ\left(h^{\prime}\right)^{n}\right|_{A^{n}}=\left.h^{\prime} \circ \pi_{S}\right|_{A^{n}}=h^{\prime} \circ \phi(\pi)_{A},
$$

where $T=\mathscr{M}(B ; n, n ; Q)$. Whence $\phi(\pi) \in \bar{\Omega}_{m} \mathbf{G}$.

It is straightforward to check that $\phi$ is a semigroup homomorphism. As for continuity, given any homomorphism $\alpha: \bar{\Omega}_{m} \mathbf{G} \rightarrow G$ with $G \in \mathbf{G}$, the composite $\alpha \circ \phi: \bar{\Omega}_{n} \mathbf{C S} \rightarrow G$ is a homomorphism and, therefore, it is continuous since $G \in \mathrm{CS}$. Hence $\phi$ is continuous.

Next, consider the mapping

$$
\psi: \bar{\Omega}_{m} \mathbf{G} \rightarrow \bar{\Omega}_{n} \mathbf{C S}
$$

defined by

$$
\psi(\pi)_{s}\left(s_{1}, \ldots, s_{n}\right)=\pi_{G}\left[g_{1}, \ldots, g_{n},\left(g_{i j}\right)\right]
$$

for $S \in \mathrm{CS}$ and $s_{1}, \ldots, s_{n} \in S$ where $G$ denotes the $\mathscr{H}$-class of $s_{1}$, and $g_{k}, g_{i j} \in G$ are given by

$$
\begin{aligned}
& s_{k}=\left(s_{k} s_{1}\right)^{\omega} g_{k}\left(s_{1} s_{k}\right)^{\omega} \quad(k=1, \ldots, n) \\
& g_{i j}=\left(s_{1} s_{i}\right)^{\omega}\left(s_{j} s_{1}\right)^{\omega} \quad \cdot(i, j=2, \ldots, n) .
\end{aligned}
$$

Lemma 3.2. The mapping $\psi$ is a well-defined continuous homomorphism.

Proof. Let $\theta: S \rightarrow T$ be a homomorphism with $S, T \in \mathbf{C S}$ and consider elements $s_{1}, \ldots, s_{n} \in S$ together with the associated $g_{k}, g_{i j}$ given by (1) and (2). Then $\theta$ maps the $\mathscr{H}$-class $G$ of $s_{1}$ into the $\mathscr{H}$-class $H$ of $\theta\left(s_{1}\right)$. It follows that the parameters $h_{k}, h_{i j}$ corresponding to $\theta\left(s_{1}\right), \ldots, \theta\left(s_{n}\right)$ according to (1) and (2) are precisely $\theta\left(g_{k}\right), \theta\left(g_{i j}\right)$ respectively. Hence

$$
\begin{aligned}
\psi(\pi)_{T}\left[\theta\left(s_{1}\right), \ldots, \theta\left(s_{n}\right)\right] & =\pi_{H}\left[h_{1}, \ldots, h_{n},\left(h_{i j}\right)\right] \\
& =\theta\left[\pi_{G}\left[g_{1}, \ldots, g_{n},\left(g_{i j}\right)\right]\right] \text { since } \pi \in \Omega_{n} \mathbf{C S} \\
& \left.=\theta \psi(\pi)_{S}\left(s_{1}, \ldots, s_{n}\right)\right] .
\end{aligned}
$$


Thus, we indeed have $\psi(\pi) \in \bar{\Omega}_{n}$ CS. Moreover,

$$
\psi(\pi)=x_{1}^{\omega} \psi(\pi) x_{1}^{\omega}
$$

since $\psi(\pi)_{S}\left(s_{1}, \ldots, s_{n}\right)$ belongs to the $\mathscr{H}$-class of $s_{1}$, and so $\psi$ assumes all its values in the $\mathscr{H}$-class of $x_{1}$.

Again $\psi$ is easily seen to be a homomorphism. To show that $\psi$ is continuous, let $\beta: \bar{\Omega}_{n} \mathbf{C S} \rightarrow S$ be any homomorphism. Then $\beta \circ \psi: \bar{\Omega}_{m} \mathbf{G} \rightarrow S$ is a homomorphism defined on a group, so that its image is in G. Hence $\beta \circ \psi$ is continuous. Whence $\psi$ is continuous.

We claim that $\phi$ restricted to the $\mathscr{H}$-class of $x_{1}$ and $\psi$ are mutually inverse isomorphisms. To establish this, consider first an arbitrary $\pi \in \bar{\Omega}_{n} \mathbf{C S}$ in the $\mathscr{H}$-class of $x_{1}$. Then, for $S \in \mathrm{CS}$ and $s_{1}, \ldots, s_{n} \in S$,

$$
\begin{aligned}
(\psi \phi \pi)_{S}\left(s_{1}, \ldots, s_{n}\right) & =(\phi \pi)_{G}\left[g_{1}, \ldots, g_{n},\left(g_{i j}\right)\right] \\
& =\pi_{S}\left(s_{1}, \ldots, s_{n}\right),
\end{aligned}
$$

where $G$ denotes the $\mathscr{H}$-class of $s_{1}$ and the $g$ 's are as in (1) and (2), since the subsemigroup of $S$ generated by $\left\{s_{1}, \ldots, s_{n}\right\}$ is a homomorphic image of a subsemigroup of $\mathscr{M}(G ; n, n ; P)$ with $P$ as before, by the Rees-Suschkewitsch theorem.

On the other hand, if $\pi \in \bar{\Omega}_{n} \mathbf{G}, G \in \mathbf{G}, g_{k}, g_{i j} \in G$, and we take $S=\mathscr{M}(G ; n, n ; P)$ with $P$ as before, then

$$
\begin{aligned}
(\phi \psi \pi)_{G}\left[g_{1}, \ldots, g_{n},\left(g_{i j}\right)\right] & =(\psi \pi)_{S}\left[\left(1, g_{1}, 1\right), \ldots,\left(n, g_{n}, n\right)\right] \\
& =\pi_{G}\left[g_{1}, \ldots, g_{n},\left(g_{i j}\right)\right]
\end{aligned}
$$

where we identify $g \in G$ with $(1, g, 1)$, so that

$$
\left[\left(1, g_{1}, 1\right)\left(i, g_{i}, i\right)\right]^{\omega}\left[\left(j, g_{j}, j\right)\left(1, g_{1}, 1\right)\right]^{\omega}=(1,1, i)(j, 1,1)=\left(1, g_{i j}, 1\right)=g_{i j}
$$

and

$$
\left[\left(i, g_{i}, i\right)\left(1, g_{1}, 1\right)\right]^{\omega}\left(1, g_{i}, 1\right)\left[\left(1, g_{1}, 1\right)\left(i, g_{i}, i\right)\right]^{\omega}=(i, 1,1)\left(1, g_{i}, 1\right)(1,1, i)=\left(i, g_{i}, i\right)
$$

In view of the Rees-Suschkewitsch theorem, we have established the following:

Theorem 3.3. $\bar{\Omega}_{n} \mathbf{C S} \simeq \mathscr{M}\left(\bar{\Omega}_{m} \mathbf{G} ; n, n ; P\right)$ as topological semigroups, where $P$ denotes the matrix obtained from $\left(y_{i j}\right)$ by addition of a first row and a first column of 1 's.

We next spell out what the isomorphism in Theorem 3.3 does to a word $w \in \bar{\Omega}_{n} \mathbf{C S}$. Denote by $\theta$ the isomorphism $\bar{\Omega}_{n} \mathbf{C S} \rightarrow \mathscr{M}\left(\bar{\Omega}_{m} \mathbf{G} ; n, n ; P\right)$ issuing from the above discussion. Write $\theta(w)=(i(w), \gamma(w), t(w))$. Then $i(w)$ and $t(w)$ locate respectively the $\mathscr{R}$ - and $\mathscr{L}$-class of $w$ and so they are the indices of the first and last letter in $w$. The description of the 
group component is a bit more complicated. In general, we have the following expression:

$$
\gamma(w)=\phi\left(\left[x_{1}\left(x_{i} x_{1}\right)^{\omega}\right]^{\omega-1} x_{1} w x_{1}\left[\left(x_{1} x_{j}\right)^{\omega} x_{1}\right]^{\omega-1}\right)
$$

where $i=t(w)$ and $j=i(w)$ and $\phi$ was defined above. In particular, if $w$ lies in the $\mathscr{H}$-class of $x_{1}$, i.e., if it starts and ends with $x_{1}$, then $\gamma(w)=\phi(w)$. Moreover, in view of the definition of $\phi, \phi(w)=\phi_{11}(w)$ where $\phi_{a b}(w)$ is the word obtained from $w$ by inserting $y_{i j}$ between $x_{i}$ and $x_{j}$ whenever an occurrence of $x_{i}$ is followed by an occurrence of $x_{j}$ in $w$ with $i \neq a$ and $j \neq b$. For example, $\phi\left(x_{2}^{2} x_{1} x_{3} x_{2}\right)=x_{2} y_{22} x_{2} x_{1} x_{3} y_{32} x_{2}$.

In the above discussion we singled out the $\mathscr{H}$-class of $x_{1}$. But the same argument applies to any other $\mathscr{H}$-class. For the $\mathscr{H}$-class $H_{a b}$ of the element $x_{a} x_{b}$, the isomorphism

$$
\bar{\Omega}_{n} \mathrm{CS} \rightarrow \mathscr{M}\left(\bar{\Omega}_{m} \mathrm{G} ; n, n ; P_{a b}\right)
$$

is given by a formula analogous to (3) with the mapping $\phi_{a b}$ instead of $\phi$ and the matrix $P_{a b}$ normalized so as to have row $a$ and column $b$ filled with 1's.

Corollary 3.4. A language $L \subseteq \Omega_{n} \mathrm{CS}$ is CS-recognizable if and only if the language $\phi_{i j}\left(L_{i j}\right) \subseteq \Omega_{m} \mathbf{G}$ is $\mathbf{G}$-recognizable for each $i, j \in\{1, \ldots, n\}$ where $L_{i j}$ is the set of words in $L$ which start with $x_{i}$ and end with $x_{j}$.

Proof. By Theorem 3.3, each $H_{i j}$ is clopen. Hence a subset of $\bar{\Omega}_{n} \mathrm{CS}$ is clopen if and only if its intersection with each $H_{i j}$ is clopen. Since each mapping $\phi_{i j}: \Omega_{n} \mathbf{C S} \rightarrow \Omega_{m} \mathbf{G}$ extends to a continuous homomorphism $\bar{\Omega}_{n} \mathbf{C S} \rightarrow \bar{\Omega}_{m} \mathbf{G}$ which, restricted to $H_{i j}$, is an onto isomorphism of topological semigroups, $K \subseteq \bar{\Omega}_{n}$ CS is clopen if and only if each $\phi_{i j}\left(K \cap H_{i j}\right) \subseteq \bar{\Omega}_{m} \mathbf{G}$ is clopen. Finally, since $\phi_{i j}$ maps $\Omega_{n} \mathbf{C S}$ into $\Omega_{m} \mathbf{G}$, it suffices to apply the characterization of $V$-recognizability quoted in Section 2 , noting that, if $L=$ $K \cap \Omega_{n} \mathrm{CS}$, then $L_{i j}=K \cap H_{i j}$.

If one wishes to make the language transformations $\phi_{i j}$ more symmetrical-at the expense of dealing with more variables-one just observes that the type of construction in this section also yields an embedding (as a closed subsemigroup)

$$
\bar{\Omega}_{n} \operatorname{CS} \hookrightarrow \mathscr{M}\left(\bar{\Omega}_{n+n^{2}} \mathrm{G} ; n, n ;\left(y_{i j}\right)\right)
$$

for which the image of $w \in \Omega_{n}$ CS which starts and ends with $x_{1}$ has group component the word $\psi(w)$ obtained from $w$ by inserting $y_{i j}$ between $x_{i}$ and $x_{j}$ for any $i, j \in\{1, \ldots, n\}$. Hence, if $L \subseteq \Omega_{n} C S$ is such that every word in $L$ starts and ends with $x_{1}$ and $\psi(L) \subseteq \Omega_{n+n^{2}} \mathbf{G}$ is $\mathbf{G}$-recognizable, then $L$ is CS-recognizable.

\section{Join-irreducibility of CS}

By the already mentioned theorem of Reiterman [20], sub-pseudovarieties of a given 
pseudovariety $\mathbf{V}$ are defined by formal equalities between implicit operations on $\mathbf{V}$. Thus, knowledge of the structure of $\bar{\Omega}_{n} \mathrm{CS}$ should lead to information about the lattice of sub-pseudovarieties of CS. In this section we indicate an application of Theorem 3.3 of this type, by considering the join-irreducibility of CS in that lattice. Since the structure theorem reduced the description of $\bar{\Omega}_{n} \operatorname{CS}$ to the study of $\bar{\Omega}_{m} \mathbf{G}$, it is natural to expect that the work of this section will translate the join-irreducibility of CS to a property of the groups $\bar{\Omega}_{n} \mathbf{G}$.

As a starting observation, we note that $\mathbf{G}$ is join-irreducible. To prove this assertion, we first point out that one may embed the symmetric group $S_{n}$ in the alternating group $A_{2 n}$, for example by sending $\sigma \in S_{n}$ to the permutation $\sigma^{\prime}$ such that $\sigma^{\prime} i=\sigma i$ if $i \leqq n$ and $\sigma^{\prime} i=n+\sigma(i-n)$ if $i>n$. Hence any infinite set of finite alternating groups generates $\mathbf{G}$. Now, if $\mathbf{G} \subseteq \mathbf{V} \vee \mathbf{W}$, then, for each $n \geqq 5, A_{n} \in \mathbf{V} \vee \mathbf{W}$. But $A_{n}$ is a simple group, and so it follows that $A_{n} \in \mathrm{V}$ or $A_{n} \in \mathrm{W}$ (for a much stronger statement, see [12, Th. V.11.1]). Hence $\mathbf{G} \subseteq \mathbf{V}$ or $\mathbf{G} \subseteq \mathbf{W}$.

Let $\mathbf{V}$ be any pseudovariety of finite simple semigroups. Consider the associated congruence

$$
\theta_{n, \mathrm{~V}}=\left\{(\pi, \rho) \in \bar{\Omega}_{n} \mathrm{CS} \times \bar{\Omega}_{n} \mathrm{CS}: \mathrm{V} \models \pi=\rho\right\}
$$

This is a closed fully invariant congruence on $\bar{\Omega}_{n} \mathrm{CS}$ (see [2] for further properties). Let $H$ be a maximal subgroup of $\bar{\Omega}_{n}$ CS. Then the class $H_{\mathrm{v}}$ of the idempotent $e$ of $H$ for the congruence $\theta_{n, \mathrm{v}}$ restricted to $H$ is a closed fully invariant subgroup of $H$.

Lemma 4.1. With the above notation, the following are equivalent:

(i) V satisfies no nontrivial CS-pseudoidentities in $n$ variables;

(ii) for each maximal subgroup $H$ of $\bar{\Omega}_{n} \mathbf{C S}, H_{\mathbf{v}}=\{e\}$;

(iii) for some maximal subgroup $H$ of $\bar{\Omega}_{n} \mathrm{CS}, H_{\mathrm{v}}=\{e\}$.

Proof. Clearly (i) $\Rightarrow$ (ii) and (ii) $\Rightarrow$ (iii). So, assume that $H$ is a maximal subgroup of $\bar{\Omega}_{n} \mathrm{CS}$ and $H_{\mathrm{V}}=\{e\}$.

By Green's Lemma [11], for any other maximal subgroup $K$ of $\bar{\Omega}_{n}$ CS, there are $\alpha, \beta, \gamma, \delta \in \bar{\Omega}_{n} \mathrm{CS}$ such that

$$
\begin{aligned}
& H \rightarrow K \\
& \pi \mapsto \alpha \pi \beta
\end{aligned} \quad \text { and } \quad \begin{aligned}
& K \rightarrow H \\
& \rho \mapsto \gamma \rho \delta
\end{aligned}
$$

are mutually inverse bijections. Hence we obtain (ii). Thus, if $\pi$ and $\rho$ are distinct members of $\bar{\Omega}_{n} \mathrm{CS}$ and $\mathrm{V}=\pi=\rho$, then $\pi \rho$ must lie in distinct maximal subgroups. Moreover, in this case we have $V \models \pi^{\omega}=\rho^{\omega}$, so that $\theta_{n, v}$ identifies two distinct idempotents $e$ and $f$. Suppose, for instance, that $e$ and $f$ are not $\mathscr{R}$-related. Then $e$ and $(f e)^{\omega}$ are $\theta_{n, v}$-equivalent but not $\mathscr{R}$-related. Hence., we may assume that $e \mathscr{L} f$. Using Green's Lemma as above and the fact that $\theta_{n, v}$ is fully invariant, we may further assume that $e=x_{1}^{\omega}$ and $f=\left(x_{2} x_{1}\right)^{\omega}$. Applying an endomorphism of $\bar{\Omega}_{n}$ CS which exchanges $x_{1}$ with $x_{2}$, we obtain also $x_{2}^{\omega} \theta_{n, v}\left(x_{1} x_{2}\right)^{\omega}$. Hence $\left(x_{1} x_{2}\right)^{\omega}\left(x_{2} x_{1}\right)^{\omega} \theta_{n, v} x_{2}^{\omega} x_{1}^{\omega}$ and so 
$x_{1}^{\omega} \theta_{n, \mathrm{v}}\left(x_{2} x_{1}\right)^{\omega}$. Consequently $x_{1}^{\omega} \theta_{n, \mathrm{v}}\left(x_{1} x_{2}\right)^{\omega}\left(x_{2} x_{1}\right)^{\omega}$. But $\left(x_{1} x_{2}\right)^{\omega}\left(x_{2} x_{1}\right)^{\omega}$ is one of the free generators $\left(y_{22}\right)$ of the maximal subgroup $H$ containing $x_{1}^{\omega}$ according to the identification with $\bar{\Omega}_{m} \mathbf{G}$ of Section 3 (see (2)). Hence $H_{\mathrm{v}} \neq\{e\}$, in contradiction with the hypothesis.

From hereon, take $H$ to be the maximal subgroup of $\bar{\Omega}_{n} \operatorname{CS}$ containing $x_{1}$, where $n$ is given by the context.

By Reiterman's theorem [20], if $\mathbf{V}$ is a proper sub-pseudovariety of $\mathbf{C S}$, then $\mathbf{V}$ satisfies some nontrivial CS-pseudoidentity, say in $n$ variables. Thus $H_{\mathrm{v}} \neq\{e\}$. If $\mathrm{W}$ is another proper sub-pseudovariety of $\mathrm{CS}$, we may change $n$ so that also $H_{\mathrm{w}} \neq\{e\}$. Since $\theta_{n, v \vee w}=\theta_{n, \mathbf{v}} \cap \theta_{n, \mathbf{w}}$, we have $H_{\mathrm{v} \vee \mathrm{w}}=H_{\mathbf{v}} \cap H_{\mathbf{w}}$. So, to conclude that $\mathbf{v} \vee \mathbf{W} \neq \mathbf{C S}$ it would suffice to show that the intersection of two nontrivial closed fully invariant subgroups of $\bar{\Omega}_{n} \mathbf{G}$ is again nontrivial.

The corresponding property for free groups is certainly true. Indeed, suppose that $N$ and $K$ are nontrivial normal subgroups of a free group $F$ such that $N \cap K=\{1\}$. Then, for $1 \neq a \in N$ and $1 \neq b \in K, a b a^{-1} b^{-1} \in N \cap K$ and so $a$ and $b$ commute. Thus, the subgroup generated by $\{a, b\}$ is a nontrivial abelian subgroup of $F$. Since it is free by Schreier's theorem, it must be cyclic. Hence there are integers $k$ and $l$ such that $a^{k}=b^{l} \neq 1$, in contradiction with $N \cap K=\{1\}$.

However, our proposed property for $\bar{\Omega}_{m} \mathbf{G}$ fails for $m=1$.

Example 4.2. For a prime $p$, let $\mathbf{G}_{p}$ denote the class of all finite groups without elements of order $p$. Let

$$
N_{p}=\left\{\pi \in \bar{\Omega}_{1} \mathbf{G}: \mathbf{G}_{p} \models \pi=1\right\}
$$

By [6], $N_{p}$ is a nontrivial subgroup of $\bar{\Omega}_{1}$ G. If $p$ and $q$ are distinct primes, clearly the pseudovariety $\mathbf{H}=\mathbf{G}_{p} \vee \mathbf{G}_{q}$ contains all finite cyclic groups and so $\mathbf{H}$ satisfies no nontrivial pseudoidentity in one variable. Hence $N_{p} \cap N_{q}=\{1\}$. However, as we already observed, $\mathbf{H} \neq \mathbf{G}$ and indeed $\mathbf{H}$ satisfies nontrivial $\mathbf{G}$-pseudoidentities in two variables.

Nevertheless, we have the following:

Theorem 4.3. For $m \geqq 2$, any two nontrivial closed normal subgroups of $\Omega_{m} \mathbf{G}$ have a nontrivial intersection.

This result was conjectured by the author based, in part, on the above discussion. It has since been established by $\mathbf{M}$. Jarden in a private communication. A proof of Theorem 4.3 is included in [5]; it is based on Jarden's proof but avoids getting too involved in the theory of profinite groups [15].

We sum up the discussion in this section in the following:

Theorem 4.4. The pseudovariety $\mathrm{CS}$ is join-irreducible.

Proof. First, if $\mathbf{V}$ and $\mathbf{W}$ are sub-pseudovarieties of $\mathrm{CS}$ satisfying nontrivial 
CS-pseudoidentities in $n \geqq 2$ variables, then $\mathbf{V} \vee \mathbf{W}$ also satisfies a nontrivial CSpseudoidentity in $n$ variables. To prove this, it suffices to use Lemma 4.1 and Theorem 4.3.

Next observe that, for any two pseudovarieties $\mathbf{V}$ and $\mathbf{W}$ of semigroups,

$$
(V \vee W) \cap C S=(V \cap C S) \vee(W \cap C S)
$$

Indeed, for the nontrivial inclusion ( $\subseteq$ ), (suppose $S_{1} \in \mathbf{V}, S_{2} \in \mathbf{W}, U$ is a subsemigroup of $S_{1} \times S_{2}$ and $T \in \mathbf{C S}$ is a homomorphic image of $U$. Then $T$ is also a homomorphic image of the minimal ideal of $U$, and so we may assume $U \in \mathbf{C S}$ and, therefore, we may assume $S_{1}, S_{2} \in C S$. Hence $C S$ is join-irreducible in the lattice of pseudovarieties of semigroups.

The group $\bar{\Omega}_{m} \mathbf{G}$ is obtained by completion of the free group on $m$ generators with respect to the initial topology for the homomorphisms into finite groups [4]. This topology on free groups was introduced by $M$. Hall [16] and has recently been used by Pin [19] in connection with a problem proposed by Rhodes [21]. The group $\bar{\Omega}_{m} \mathbf{G}$ is also a free profinite group, and it is based on known properties of such groups that Jarden obtained his proof of Theorem 4.3.

Acknowledgements. I would like to express my thanks to Assis Azevedo, Mario Petrich and Norman Reilly for helpful discussions during the preparation of this paper. I am particularly indebted to Moshe Jarden for establishing Theorem 4.3.

\section{REFERENCES}

1. J. Almeida, The algebra of implicit operations, Algebra Universalis 26 (1989), 16-32.

2. J. Almeida, On pseudovarieties, varieties of languages, filters of congruences, pseudoidentities and related topics, Algebra Universalis 27 (1990), 333-350.

3. J. Almeida, Residually finite congruences and quasi-regular subsets in uniform algebras, Portugal. Math. 46 (1989), 313-328.

4. J. Almeida, Equations for pseudovarieties, in J.-E. Pin (Ed.), Formal Properties of Finite Automata and Applications, (Lecture Notes in Comput. Sci. 386, Springer-Verlag, 1989), 148-164.

5. J. Almeida, Recent developments in the theory of implicit operations (Proceedings of the 1989 Berkeley Workshop on Monoids), to appear.

6. J. Almeida and A. Azevedo, Implicit operations on certain classes of semigroups, in S. Goberstein and P. Higgins (Eds.), Semigroups and their Applications (Proceedings 1986 Chico Conf., D. Reidel, 1987), 1-11.

7. C. J. Ash, Pseudovarieties, generalized varieties and similarly described classes, J. Algebra 92 (1985), 104-115.

8. B. BANASCHEWSKı, The Birkhoff Theorem for varieties of finite algebras, Algebra Universalis 17 (1983), 360-368.

9. S. Burris and H. P. Sankappanavar, A Course in Universal Algebra (Springer-Verlag, New York, 1981).

10. A. H. Clifford, The free completely regular semigroup on a set, J. Algebra 59 (1979), 434-451. 
11. A. H. Clifford and G. B. Preston, The Algebraic Theory of Semigroups, Vol. 1 (Amer. Math. Soc., Providence, 1961).

12. S. Ellenberg, Automata, Languages and Machines, Vol. B (Academic Press, New York, 1976).

13. S. Eilenberg and M. P. Schótzenberger, On pseudovarieties, Adv. in Math. 19 (1976), 413-418.

14. T. Evans, Varieties of semigroups, Semigroup Forum 2 (1971), 1-43.

15. M. D. Fried, and M. JARDEN, Field Arithmetic (Springer-Verlag, Berlin, 1986).

16. M. Hall $\mathrm{J}_{\mathrm{R}}$., A topology for free groups and related groups, Ann. of Math. 52 (1950), $127-139$.

17. G. Lallement, Semigroups and Combinatorial Applications (Wiley, New York, 1979).

18. J.-E. PIN, Varieties of Formal Languages (Plenum, London, 1986).

19. J.-E. PIN, Topologies for the free monoid (LITP report 88-17), (to appear in J. Algebra).

20. J. Reiterman, The Birkhoff theorem for finite algebras, Algebra Universalis 14 (1982), 1-10.

21. J. Rhodes, New techniques in global semigroup theory, in S. Goberstein and P. Higgins (Eds.), Semigroups and their Applications (Proceedings 1986 Chico Conf., D. Reidel, 1987), 169-181.

Inic-Centro de Matemätica

FACULDADE de ClêNCIAS

Universidade do Porto

4000 PORTO

Portugal 